\title{
Correction to: Dynamically evolving algorithm for minimizing the energy consumption of a manipulator
}

\section{Yoshio Yokose $^{1}$}

Published online: 5 December 2018

(C) International Society of Artificial Life and Robotics (ISAROB) 2018

\section{Correction to: Artificial Life and Robotics}

https://doi.org/10.1007/s10015-018-0487-1

In the original publication of the article, the article title was incorrectly published as "Dynamically evolutional algorithm for minimization of the consumption energy of a manipulator".

The correct title is "Dynamically Evolving Algorithm for Minimizing the Energy Consumption of a Manipulator".

The original article has been updated.

The original article can be found online at https://doi.org/10.1007/ s10015-018-0487-1.

Yoshio Yokose

yokose@kure-nct.ac.jp

1 Department of Electrical Engineering and Information

Science, National Institute of Technology, Kure College,

2-2-11 Agaminami, Kure, Hiroshima 737-8506, Japan 